\title{
Interferencia del receptor del factor de crecimiento epidermoide (EGFR) y su utilidad como adyuvante en el tratamiento del cáncer avanzado de cabeza y cuello
}

\author{
Interference of epidermal growth factor receptor (EGFR) \\ and its usefulness in the treatment of advanced head and neck cancer
}

\author{
Pedro Cortez V'1 Mariela Torrente A ${ }^{1}$.
}

\begin{abstract}
RESUMEN
El receptor del factor de crecimiento epidermoide (EGFR) se encuentra sobreexpresado en más del $90 \%$ de los tumores escamosos de cabeza y cuello. Se han desarrollado diversos métodos para interferir con el EGFR entre los cuales el más utilizado es el anticuerpo monoclonal cetuximab. En esta revisión se discuten los resultados disponibles a la fecha del uso de cetuximab como adyuvante al tratamiento de pacientes con carcinomas avanzados de cabeza y cuello.
\end{abstract}

Palabras claves: Cáncer de cabeza y cuello, factor de crecimiento epidermoide, cetuximab.

\begin{abstract}
Epidermal growth factor receptor is overexpressed in more than $90 \%$ of the cases of head and neck squamous cell carcinoma (SCCHN). The addition of the monoclonal antibody cetuximab to therapy in patients with advanced SCCHN is discussed in this review.

Key words: Head and neck squamous cell carcinoma, epidermal growth factor receptor, cetuximab.
\end{abstract}

1 Médico Servicio de Otorrinolaringología, Hospital Clínico Universidad de Chile. 


\section{INTRODUCCIÓN}

El cáncer de cabeza y cuello es la sexta neoplasia maligna más común en el mundo. Aproximadamente el 90\%-95\% son carcinomas de células escamosas (CECC), y la mayoría de Ios pacientes $(60 \%)$ se presentan en etapa III-IV localmente avanzadas². En Chile no existen estudios de prevalencia actualizados de cáncer de cabeza y cuello, pero se sabe que la tasa de mortalidad anual por esta causa en 2003 corresponde a 1,65 por $100.000 \mathrm{hab}^{3}$. A pesar de los avances en el conocimiento de los mecanismos oncogénicos y de las modalidades terapéuticas, la sobrevida de estos pacientes no ha mejorado en forma significativa en los últimos 20 años ${ }^{2}$. Cincuenta a sesenta por ciento de los pacientes con CECC localmente avanzado presentan recurrencia locorregional en su mayoría antes de los dos años de evolución, y el 20\%-30\% desarrollan metástasis a distancia ${ }^{4}$.

En los últimos 10 años se ha realizado un cambio terapéutico con la finalidad de preservar el órgano comprometido, esto por las secuelas funcionales de la cirugía resectiva. Es así como se han desarrollado protocolos de radio-quimioterapia en distintas modalidades de administración y de drogas. Las combinaciones más utilizadas son administración secuencial de un esquema que contenga un taxano (Paclitaxel/Docetaxel) seguido de radioterapia o uso concomitante de cisplatino y radioterapia.

Entre las alteraciones moleculares más frecuentes en estos tumores, está la sobreexpresión del receptor del factor de crecimiento epidermoide (EGFR), lo que se ha identificado en más del $90 \%$ de los casos estudiados ${ }^{5,6}$. Esto impulsó el estudio y desarrollo de moléculas de interferencia que permitieran mejorar la sobrevida de pacientes en estadios avanzados de enfermedad. El objetivo de la presente revisión es describir las terapias disponibles a la fecha que interfieren con la activación del EGFR y cuál ha sido su impacto en la sobrevida de los pacientes con estadios avanzados de enfermedad.

\section{BIOLOGÍA DEL EGFR}

El receptor del factor de crecimiento epidermoide es una familia de proteínas transmembrana que forma parte de los receptores tirosín-kinasa, es decir, su dominio intracelular al ser activado tiene la capacidad de fosforilar proteínas. En este grupo se reconocen 4 integrantes: EGFR (ErbB1, HER1), ErbB2 (HER2), ErbB3 (HER3) y ErbB4 (HER4) ${ }^{7}$. La activación de EGFR por mediadores extracelulares tales como factor de crecimiento epidermoide (EGF), TGFa y amfiregulina, producen un cambio conformacional que induce la autofosforilación de la porción intracelular. Esto a su vez tiene un efecto de amplificador en vías tales como Ras/ MAPK (estimulación de proliferación y migración) y PI3K/Akt (estimulación de crecimiento celular, proliferación y migración) ${ }^{8}$.

El EGFR se sobreexpresa en más del $90 \%$ de los casos de carcinoma escamoso de cabeza y cueIlo, y esto se relaciona con un peor pronóstico $0^{6,9}$. Se pueden utilizar distintas alternativas como complemento al tratamiento: vacunas anti-EGFR, transporte de citotóxicos ligados a EGFR, inhibición de la actividad de kinasa mediante anticuerpos 0 moléculas de interferencia ${ }^{9}$. Actualmente el uso de moléculas de interferencia como geftinib (Iressa ${ }^{\mathrm{TM}}$ ) o erlotinib (Tarceva ${ }^{\mathrm{TM}}$ ) se encuentra aprobado para su uso en carcinoma de pulmón de células pequeñas ${ }^{10,11}$. Diversos estudios en fase II con pacientes portadores de carcinoma de cabeza y cuello se han reportado, pero el uso de estas moléculas aún se encuentra en fase de investigación ${ }^{12-14}$.

Cetuximab (Erbitux ${ }^{\mathrm{TM}}$ ) es un anticuerpo monoclonal anti EGFR. Su uso en pacientes con CECC ha sido aprobado en EE.UU (FDA) como adyuvante a la radio y/o quimioterapia. El costo en Chile de asociar Erbitux al tratamiento de un paciente es de $\$ 1,2$ a $\$ 1,8$ millones semanales adicionales ${ }^{15}$. Desde que en el año 2006 se publicó el primer estudio utilizando este producto ${ }^{16}$, se han reportado diversos estudios Ios cuales se discuten a continuación. Los mecanismos de acción mediados por cetuximab serían por dos vías: uniéndose al EGFR de manera directa, inhibiendo la unión del ligando y la fosforilación y la otra vía, mediante la inducción de citotoxicidad celular dependiente de anticuerpos (ADCC), en consecuencia, la destrucción de las células recubiertas de anticuerpos por el sistema inmune del huésped ${ }^{17}$. Si bien los efectos anticancerígenos de cetuximab a través de la inhibición de la transducción de señales han sido demostrados ${ }^{18,19}$, la contribución de la ADCC aún se encuentra en estudio. 


\section{CETUXIMAB Y QUIMIORRADIOTERAPIA SECUENCIAL (QUIMIOTERAPIA DE INDUCCIÓN)}

El uso del "enfoque secuencial", mediante el cual la quimioterapia es administrada antes de la radioterapia, o quimiorradioterapia (QR) neoadyuvante, es uno de los esquemas terapéuticos que se utiliza en pacientes con enfermedad localmente avanzada y/o irresecable.

En el ensayo clínico Extreme ${ }^{20}$ se investigó el beneficio de agregar cetuximab a la quimioterapia. Fue el primer ensayo fase III en demostrar una mejoría significativa en la sobrevida global desde la introducción del cisplatino en el tratamiento del CECC localmente avanzado. Dicho estudio incluyó a 442 pacientes con diagnóstico de CECC recurrente y/o metastásico y los randomizó en grupos de tratamiento con cisplatino o carboplatino más 5-fluoruracilo asociado o no a cetuximab. La adición de cetuximab aumentó la tasa de respuesta global de $20 \%$ a $36 \%$ (p 0,001). El promedio de sobrevida libre de progresión de enfermedad aumentó de 3,3 a 5,6 meses ( $p$ 0.001) y el promedio de sobrevida global de 7,1 a 10,4 meses ( $p 0,04$ ). Otro importante hallazgo en este estudio fue que el grupo de cetuximab no presentó mayor tasa de toxicidad específica asociado a quimioterapia. Solo existió mayor tasa de hipomagnesemia y sepsis, respecto del grupo con solo quimioterapia, pero no aumentó la mortalidad asociada a la droga. Esto es de suma importancia en aquellos esquemas secuenciales con quimioterapia de inducción.

El estudio fase III ECOG 5397, con 117 pacientes, randomizó grupos a recibir cisplatino más placebo vs cisplatino más cetuximab ${ }^{21}$. Este último grupo presentó aumento en la respuesta global de $10 \%$ a $26 \%$ ( $p$ 0,03) pero no hubo mejoría significativa en la sobrevida libre de enfermedad 0 sobrevida global.

Los protocolos previamente mencionados no incluían taxanos en su esquema de quimioterapia, lo cual ha demostrado tener mejor respuesta global versus sólo cisplatino y fluoracilo ${ }^{22,23}$. La limitante de esta asociación es la toxicidad (hemorragia gastrointestinal, diarrea y neutropenia febril). Hitt y cols $^{24}$ reportan un ensayo clínico fase II, en que se incluyó a 46 pacientes con diagnóstico de CECC recurrente/metastásico y que utilizó Paclitaxel combinado con cetuximab con una tasa de respuesta global de $54 \%$, promedio de sobrevida libre de progresión de 4,2 meses y tasa de sobrevida global de 8,1 meses, comparable con controles históricos. La tasa de respuesta local al combinar Paclitaxel con cetuximab fue más alta que la demostrada por el estudio EXTREME al utilizar Cisplatino/5-FU con o sin cetuximab (36 vs $20 \%$ respectivamente), sin embargo los promedios de sobrevida libre de progresión y sobrevida global son similares en ambos regímenes. En general, la tolerabilidad de este régimen de tratamiento, del grupo de Hitt, fue aceptable en comparación al estudio EXTREME, destacando la presentación de rash cutáneo (24\%), astenia (17\%), neutropenia $(13 \%)$ y mucositis (7\%), entre otras. Los 12 pacientes que debieron suspender la terapia debido a efectos adversos, completaron en promedio al menos 6 meses de tratamiento efectivo.

La Asociación Francesa de Oncología y Radioterapia de Cabeza y Cuello (GORTEC) ${ }^{25}$ publicó un estudio con esquema secuencial que incluyó a 153 pacientes con carcinoma escamoso de laringe/ hipofaringe en etapa III y IV. Los pacientes recibían 3 cursos de quimioterapia de inducción y luego se randomizaban en dos grupos: radioterapiacisplatino vs radioterapia-cetuximab. En ambos grupos no hubo diferencia con respecto a la tasa de preservación de órgano, pero en el grupo de radioterapia-cetuximab hubo mejor tolerancia y menor tasa de toxicidad. La sobrevida a 18 meses no mostró diferencias significativas ( $92 \%$ y $89 \%$ respectivamente).

El ensayo clínico del grupo norteamericano ECOG E2303 (Eastern Cooperative Oncology Group ${ }^{26}$ describe el uso de cetuximab asociado a un régimen de inducción de carboplatino-Paclitaxel en 63 pacientes con enfermedad resecable etapa III/IV (21/42 pacientes respectivamente, siendo de orofaringe $61 \%$, laringe $22 \%$ y otros $16 \%$ ). El control de la enfermedad primaria a los 2 años fue del $83 \%$. La sobrevida general a 1 año y 2 años fue de $97 \%$ y $82 \%$; la sobrevida libre de progresión fue de $82 \%$ y $66 \%$ respectivamente con una tasa de preservación de órgano de $91 \%$.

Mesía y cols evalúan la eficacia y seguridad de cetuximab como tratamiento de inducción antes de radioterapia definitiva más cetuximab concomitante en pacientes con cáncer de orofaringe localmente avanzado ${ }^{27}$. Este ensayo clínico fase II 
logró determinar que el control locorregional de la enfermedad al año de sobrevida fue superior en el grupo con cetuximab de inducción ( $59 \%$ vs $47 \%$ ), pero este resultado no se mantuvo a los 2 años de sobrevida. La toxicidad fue similar en ambos grupos siendo principalmente a nivel de la piel.

Tomados en grupo, los resultados de distintos ensayos clínicos que emplean cetuximab en el tratamiento de inducción, demuestran algunas mejoras en términos de tazas de respuesta, pero requiere de estudios aleatorios más exhaustivos ${ }^{28}$. La adición de cetuximab a la terapia de inducción no está libre de inconvenientes: el impacto de las toxicidades específicas a drogas, tales como reacciones alérgicas a la infusión, hipomagnesemia, erupciones en la piel y dermatitis generalizada puede reducir el cumplimiento del tratamiento en los tiempos adecuados. También toxicidades específicas de la quimioterapia, como neutropenia, parecen estar aumentadas por el cetuximab ${ }^{29}$.

\section{CETUXIMAB Y QUIMIORRADIOTERAPIA CONCOMITANTE}

La administración concomitante de quimioterapia y radiación ha demostrado ser la opción estándar para tratar los pacientes con CECC localmente avanzado ${ }^{30}$. Incluso, esta combinación ha logrado mejorar los resultados cuando se utiliza como tratamiento de preservación de órganos en cáncer de laringe e hipofaringe irresecable y en el tratamiento adyuvante, después de cirugía resectiva ${ }^{28}$. Sin embargo, el aumento en la sobrevida global de los pacientes ha sido menor de lo esperado (cercano al 5\%), por lo cual se siguen explorando alternativas terapéuticas. El interés en la adición de cetuximab a los regímenes de la QR se deriva de la demostración de una mejoría en la supervivencia cuando se añade a la radioterapia sola, lo cual apoya el papel del cetuximab como agente citotóxico per $s e^{16}$; el beneficio demostrado cuando cetuximab fue probado en combinación con quimioterapia en la enfermedad recurrente/ metastásica ${ }^{20}$; y no aumenta la toxicidad de la radioterapia en forma significativa ${ }^{16}$.

En el año 2006, Pfister y cols publican el primer estudio que incluía cetuximab con $Q R$ concomitante $^{31}$. El estudio incluyó a 21 pacientes con CECC estadio III/IV, M0 (orofaringe 59\%, laringe 18\%, cavidad oral $14 \%)$. Se utilizó radioterapia por 6 semanas (1,8 Gy / una vez al día semana del 1 al 6; se agrega un segundo boost de 1,6 Gy/día durante la semana 5-6; 70 Gy total a la región de enfermedad macroscópica y 45 Gy a la zona del cuello) y en forma concomitante cisplatino (100 $\mathrm{mg} / \mathrm{m}^{2}$ semanas 1 y 4$)$ y cetuximab $\left(400 \mathrm{mg} / \mathrm{m}^{2}\right.$ día uno y $250 \mathrm{mg} / \mathrm{m}^{2}$ sem 2-10). A los 3 años el porcentaje de sobrevida libre de progresión de enfermedad fue de $56 \%$, control locorregional fue de $71 \%$ y sobrevida global de $76 \%$ a los 3 años. Sin embargo, el estudio debió suspenderse de forma prematura por la presentación de eventos adversos sistémicos graves (2 de tipo infecciosos y 2 cardiovasculares), sin eventos adversos locales llamativos. La contribución del cetuximab en dichos eventos adversos fue incierta.

En el año 2008, el estudio encabezado por Langer y cols $^{32}$ (ECOG 3303), evaluó la combinación de un régimen de radioterapia fraccionada $(70$ Gy/2Gy/dia $\times 7$ semanas) con cisplatino $\left(75 \mathrm{mg} / \mathrm{m}^{2}\right.$ los días 15,36 y 57$)$ y cetuximab $\left(400 \mathrm{mg} / \mathrm{m}^{2}\right.$ el día 1 y luego $250 \mathrm{mg} / \mathrm{m}^{2}$ semanales). Se incluyeron 61 pacientes con enfermedad localmente avanzada irresecable (98\% etapa IV), siendo el tumor primario de base de lengua (34\%), amígdalas (21\%) y otro sitios de orofaringe (13\%) la mayoría. La tasa de respuesta global fue del $48 \%$ y en otro $31 \%$ se logró mantener estable la enfermedad. El $74 \%$ recibió las 3 dosis de cisplatino $(94 \%$ al menos 2 dosis) y el $31 \%$ de los pacientes completó más de 6 meses de tratamiento con cetuximab. Hubo un caso de muerte debido a neutropenia febril. Entre otros efectos adversos se describió: neutropenia (26\%), mucositis oral (54\%), alteración local de la piel con reacción acneiforme (15\%). Pese a ello, el régimen de combinación se consideró factible de aplicar.

El grupo Radiation Therapy Oncology Group (RTOG) dependiente del Instituto de Salud de Estados Unidos (NIH) publicó un ensayo clínico randomizado fase III (RTOG 0522) ${ }^{33}$, que incluyó a 940 pacientes con CECC avanzado, etapas III $(14 \%)$ y IV $(86 \%)$, de orofaringe $(70 \%)$, laringe e hipofaringe. Se comparó el uso de cisplatino (100 $\mathrm{mg} / \mathrm{m}^{2}$ cada 3 semanas por 2 veces) más radioterapia (dosis total de 72 Gy en 42 fracciones durante 6 semanas) con o sin cetuximab (dosis de carga 
de $400 \mathrm{mg} / \mathrm{m}^{2}$ y luego $250 \mathrm{mg} / \mathrm{m}^{2}$ semanales). La adición de cetuximab no logró demostrar mejoras en la sobrevida libre de enfermedad $(p=0,66)$ ni en la sobrevida global $(79,7$ vs $82,6 \% p=0,17)$ a dos años de seguimiento. Además, su uso se asoció a mayor grado de mucositis ( 45 vs $35 \%, p=0,003$ ) y dermatitis (40 vs $17 \%, p=0,0001$ ).

Para Numico y cols ${ }^{28}$, las conclusiones de estos resultados deben ser interpretadas con cautela. Probablemente falta tiempo de seguimiento para poder definir conclusiones más firmes. En ninguna otra publicación aparecen tasas de sobrevida tan altas, lo que podría deberse a la mejor selección de pacientes. Sin embargo, el cetuximab pareciera no tener un impacto significativo en la eficacia de la CRT, al menos cuando la combinación de cisplatino y radioterapia hiperfraccionada es utilizada. De acuerdo a esto y basado en la información disponible hasta el momento, para algunos autores la combinación de cetuximab y QRT debería considerarse aún en investigación y no ser usado en la práctica clínica ${ }^{4}$.

\section{PREDICTORES DE RESPUESTA A CETUXIMAB}

Teniendo el cetuximab un objetivo definido como es el receptor del factor de crecimiento epidermoide, su uso podría limitarse a los pacientes con tumores sensibles. Sin embargo, hasta la fecha, la búsqueda de las características clínicas y biológicas para predecir la sensibilidad o la resistencia han sido desalentadoras. La expresión de la proteína EGFR detectada por inmunohistoquímica, la amplificación del gen (tal como se detecta por fluorescencia de hibridación in situ) y EGFRvIII (una forma truncada del gen EGFR que resulta en una variante del receptor que no responde a los efectos inhibidores de moléculas anti-EGFR), no han logrado demostrar utilidad para predecir la actividad o la resistencia de Ios CECC a cetuximab ${ }^{34}$.

\section{CONCLUSIONES}

Son múltiples los estudios que han demostrado la utilidad del cetuximab en cuanto a sobrevida libre de progresión de enfermedad y sobrevida global. Sin embargo, muchos de estos trabajos, han sido criticados por su bajo número de pacientes y la heterogeneidad de los mismos, con diversos esquemas de selección de pacientes y de tratamientos, lo que hace difícil la interpretación en conjunto de todos ellos.

La adición de cetuximab parece prometedora en ensayos clínicos con quimioterapia de inducción, en los cuales las tasas de respuesta son uniformemente más altas que controles históricos. Ahora bien, da la impresión que en estos ensayos, la quimioterapia de inducción puede actuar como un seleccionador de los pacientes que presentan buena respuesta y por este motivo tener mejores resultados.

Por otra parte, los resultados son más difíciles de interpretar cuando se considera QRT concomitante debido a las diferencias en los esquemas de quimioterapia y radiación que se han utilizado. Sin embargo, los resultados preliminares de la RTOG 0522 parecen excluir un efecto positivo de la adición de cetuximab en el régimen con cisplatino. Probablemente existan otras formas de explotar la potencial ventaja del cetuximab como agente anticanceroso, ya sea, aumentando el tiempo de exposición a este fármaco, combinación con otras drogas de quimioterapia (no cisplatino) y/o combinado con otros agentes que tengan como "target" el EGFR.

Por último, la adición de cetuximab no está exento de toxicidad anexa al uso de QRT.

Por estos motivos, el uso de cetuximab no ha tenido los resultados esperados, por lo que falta definir cuáles serán los pacientes que sean realmente beneficiados con el ingreso de éste al esquema de tratamiento basal de CECC localmente avanzado.

\section{BIBLIOGRAFÍA}

1. Shibuya K, Mathers C, Boshi-Pinto C, et al. Global and regional estimates of cancer mortality and incidence site. Results for the global burden of disease 2000. BMC Cancer 2002; 2: 37.

2. Jemal $A$, Siegel $R, X u$ J, et al. Cancer Statistics. CA Cancer J Clin 2010; 60: 277-300.

3. BéJar M, Cevo J, Romero M, Iñiguez R. Mortalidad nacional en otorrinolaringología. Rev Otorrinolaringol Cir Cabeza Cuello 2007; 67(1): 31-7. 
4. Denaro N, Russi E, Merlano M. Strategies for non-resecable Head and Neck Cancer. Current Treatment Options in Oncology 2013; 14: 492504.

5. Gildener-Leapman N, Ferris R, Bauman J. Promising systemic immunotherapies in hean and neck squamous cell carcinoma. Oral Oncol 2013; 49: 1089-96.

6. Reuter CW, Morgan MA, Eckardt A. Targeting EGFreceptor-signalling in squamous cell carcinomas of the head and neck. Br J Cancer 2007; 96: 408-16.

7. Wieduwilt MJ, Moasser MM. The epidermal growth factor receptor family: biology driving targeted therapeutics. Cell Mol Life Sci 2008; 65(10): 1566-84.

8. Wykosky J, Fenton T, Furnari F, et al. Therapeutic targeting of epidermal growth factor receptor in human cancer: successes and limitations. Chin $J$ Cancer 2011; 30 (1): 5-12.

9. Ang KK, Berkey BA, Tu X, et al. Impact of epidermal growth factor receptor expression on survival and pattern of relapse in patients with advanced head and neck carcinoma. Cancer Res 2002; 62: 7350-6.

10 http://www.accessdata.fda.gov/drugsatfda_ docs/label/2013/021743s18lbl.pdf.

11. http://www.fda.gov/Drugs/DrugSafety/ PostmarketDrugSafetylnformationforPatients andProviders/DrugSafetyInformationforHeathcareProfessionals/ucm085197.htm.

12. Bauman J, Arias-Pulido H, Lee S, Fekrazad M, et al. A phase II study of temsirolimus and erlotinib in patients with recurrent and/or metastatic, platinum-refractory head and neck squamous cell carcinoma. Oral Oncol 2013; 49(5): 461-7.

13. Martins R, Parvathaneni U, Bauman J, et al. Cisplatin and radiotherapy with or without erlotinib in locally advanced squamous cell carcinoma of the head and neck: a randomized phase II trial. J Clin Oncol 2013; 31(11): 141521.

14. Rao K, Kalapurakal S, Chalasani P, et al. A phase II study of intra-arterial cisplatin with concurrent radiation and erlotinib for locally advanced head and neck cancer. Cancer Chemother Pharmacol 2013; 72(3): 545-52.

15. http://www.merck.cl/country.cl/es/images/ preciosfarma_tcm650_29186.pdf?Version=.
16. Bonner JA, Harari PM, Giralt J, et al. Radiotherapy plus Cetuximab for Squamous-cell carcinoma of the head and neck. N Engl J Med 2006; 354: 567-68.

17. Markovic A, Chung C. Current role of EGF receptor monoclonal antibodies and tyrosine kinase inhibitors in the management of head and neck squamous cell carcinoma. Expert Rev Anticancer Ther 2012; 12(9): 1149-59.

18. Kural J, ChiKumi H, Hashimoto K, et al. Antibodydependent cellular cytotoxicity mediated by cetuximab against lung cancer cell lines. Clin Cancer Res 2007; 13(5): 1552-61.

19. LI S, Schmitz K, Jeffrey P, et Al. Structural basis for inhibition of the epidermal growth factor receptor by cetuximab. Cancer Cell 2005; 7(4): 301-11.

20. Vermorken JB, Mesia R, Rivera F, et al. Platinumbased chemotherapy plus cetuximab in head and neck cancer. N Engl J Med 2008; 359: 111627.

21. Burtness B, Goldwasser MA, Flood W, et al. Phase III randomized trial of cisplatin plus placebo compared with cisplatin plus cetuximab in metastatic/recurrent head and neck cancer: An Eastern Cooperative Oncology Group Study. J Clin Oncol 2005; 23: 8646-54.

22. Posner MR, Hershock DM, Blajman C, et al. Cisplatin and Fluoruracil alone or with docetaxel in head and neck cancer. New England Journal of Medicine 2007; 357: 1705-15.

23. Pointreau Y, Garaud P, Chapet S, et al. Randomized trial of induction chemotherapy with cisplatin and 5-fluorouracil with or without docetaxel for larynx preservation. Journal of the National Cancer Institute 2009; 101: 498-506.

24. Hitt R, Irigoyen A, Cortes-Funes H, et al. Phase II study of the combination of Cetuximab and weekly Paclitaxel in the first-line treatment of patients with recurrent and/or metastatic squamous cell carcinoma of head and neck. Annals of Oncology 2012; 23: 1016-22.

25. Lefebvre J, Pointreau Y, Rolland F, et al. Groupe Oncologie Radiotherapie Tete Et Cou (GORTEC). Induction chemotherapy followed by either chemoradiotherapy or bioradiotherapy for larynx preservation: the TREMPLIN randomized phase II study. J Clin Oncol 2013; 31(7): 853-9.

26. Wanebo HJ, Ghebremichael MS, Burtness B, ET AL. Phase II induction cetuximab (C225), 
paclitaxel $(\mathrm{P})$, and carboplatin $(\mathrm{C})$ followed by chemoradiation with C225, P, C, and RT 68-72 Gy for stage III/IV head and neck squamous cancer: primary site organ preservation and disease control at 2 years (ECOG, E2303). Journal of Clinical Oncology 2010; 28(15S): 5513.

27. Mesía R, Rueda A, Vera R, et al. Adjuvant therapy with cetuximab for locally advanced squamous cell carcinoma of the oropharynx: results from a randomized, phase II prospective trial. Annals of Oncology 2012; 24: 448-53.

28. Numico G, Franco P, Cristofano A, et al. Is the combination of Cetuximab with chemoradiotherapy regimens worthwhile in the treatment of locally advanced head and neck cancer? A review of current evidence. Critical Reviews in Oncology/Hematology 2013; 85: 112-20

29. Bourhis J, Lefebvre JL, Vermorken J. Cetuximab in the management of locoregionally advanced head and neck cancer: Expanding the treatment options? Eur Journal of Cancer 2010; (46): 197989.

30. Kurzweg T, Mockelmann N, Laban S, et al. Current treatment options for recurrent/metastatic head and neck cancer: a post-ASCO 2011 update and review of last year's literatura. Eur Arch Otorhinolaryngol 2012; 269: 2157-67.

31. Pfister DG, Su YB, Kraus DH, et al. Concurrent cetuximab, cisplatin, and concomitant boost radiotherapy for locoregionally advanced, squamous cell head and neck cancer: a pilot phase II study of a new combined-modality paradigm. Journal of Clinical Oncology 2006; 24: 1072-8.

32. Langer C, Lee J, Patel U, et al. Preliminary analysis of ECOG 3303: Concurrent radiation (RT), cisplatin (DDP) and cetuximab (C) in unresectable, locally advanced (LA) squamous cell carcinoma of the head and neck (SCCHN) Journal of Clinical Oncology 2008; 26 (15s): 6006.

33. Ang KK, Zhang QE, Rosenthal D, et al. A Randomized phase III trial (RTOG05-22) of concurrent accelerated radiation plus Cisplatin with or without Cetuximab for Stage III-IV HNSC. Journal of Clinical Oncology 2011; 25(15S): abstract 5500 (ASCO meeting).

34. Numico G, Silvestris N, Russi EG. Advances in EGFR-directed therapy in head and neck cancer. Frontiers in Bioscience 2011; S3: 454-66. 